\title{
Apple Polyphenols Influence Cholesterol Metabolism in Healthy Subjects with Relatively High Body Mass Index
}

\author{
Yoko Nagasako-Akazome ${ }^{1}$, Tomomasa Kanda ${ }^{1}$, Yasuyuki Ohtake ${ }^{1}$, \\ Hiroyuki Shimasaki ${ }^{2}$ and Tetsuyuki Kobayashi ${ }^{3}$ \\ ${ }^{1}$ Fundamental Research Laboratory, Asahi Breweries, Ltd. (1-21, Midori 1-chome, Moriya-shi, Ibaraki 302-0106, JAPAN) \\ ${ }^{2}$ Department of Health and Nutrition, University of Arts and Sciences (1288, Komagome, Iwatsuki-ku, Saitama-shi, Saitama 339-8539, \\ JAPAN) \\ ${ }^{3}$ Department of Biology, Ochanomizu University (2-1-1 Ohtsuka, Bunkyo-ku, Tokyo 112-8610, JAPAN)
}

\begin{abstract}
We performed a randomized double-blind, placebo-controlled study on moderately obese male and female subjects (71 subjects) with a body mass index ranging from 23 to 30 to evaluate the efficacy of 12-week intake of polyphenols extracted from apples and hop bract $(600 \mathrm{mg} / \mathrm{day})$. We confirmed that 12 week ingestion of polyphenol-containing capsules significantly decreased total cholesterol and LDLcholesterol levels. The effects of the apple polyphenol-containing capsules were more marked than those of the hop bract polyphenol-containing capsules. The visceral fat area and the level of adiponectin in the group administered apple polyphenols improved in comparison with the control group. Blood and physical examinations revealed on clinical problems, and no adverse reactions were observed during the ingestion period. These results demonstrate that apple polyphenols regulate fat metabolism in healthy subjects with relatively high body mass index.
\end{abstract}

Key words: apple polyphenols, total cholesterol, LDL-cholesterol, body mass index, visceral fat, adiponectin

\section{INTRODUCTION}

The recent World Health Organization (WHO) report entitled "Preventing chronic diseases: a vital investment" emphasizes the necessity of addressing on an international basis the major risks associated with chronic diseases ${ }^{11}$. The global risk of overweight/obesity as well as the high incidence of diabetes, cardiovascular diseases, and specific cancers have been recognized. Globally, about one billion people are overweight or obese whereas 850 million are underweight ${ }^{2}$.

Excessive ingestion of fat results in obesity and various lifestyle-related diseases. According to a survey, percent body fat increases with dietary lipid intake ${ }^{3}$. Fat accumulation, especially visceral fat accumulation, induces metabolic syndrome associated with insulin resistance ${ }^{4)}$, causing lifestyle-related diseases such as diabetes, hyperlipidemia, and hypertension, leading to arteriosclerosis ${ }^{5}$. In addition, several studies have reported that large amounts of physiologically active substances (adipocytokines), such as leptin, tumor necrosis factor (TNF)- $\alpha$, plasminogen activator inhibitor (PAI)-1, and adiponectin, are synthesized in accumulated adipose tissue ${ }^{6-10)}$. These substances directly contribute to various diseases. Adiponectin is a hormone secreted by adipose cells and many studies have indicated its relationship to insulin resistance $^{11-13)}$. In addition, hypoadiponectinemia is associated with the clinical phenotype of metabolic syndrome and adiponectin levels may be clinically useful for detecting an underlying metabolic disorder ${ }^{14,15}$.

Recent studies investigated tea polyphenols as functional food materials and reported their antioxidant ${ }^{16-18}$, anticancer ${ }^{19)}$, blood sugar elevation-inhibiting ${ }^{20)}$, and hypotensive effects ${ }^{21)}$. With regard to serum total cholesterolreducing action, another study confirmed the efficacy of 12-week tea catechin ingestion for borderline/mild hypercholesterolemia ${ }^{22)}$. In addition, many animal experiments

* Correspondence to: Yoko Nagasako- Akazome, Fundamental Research Laboratory, Asahi Breweries, Ltd., 1-21, Midori 1-chome, Moriyashi, Ibaraki 302-0106, JAPAN

E-mail: yoko.akazome@asahibeer.co.jp

Accepted May 23, 2007 (received for review March 5, 2007)

Journal of Oleo Science ISSN 1345-8957 print / ISSN 1347-3352 online

http://jos.jstage.jst.go.jp/en/ 
have recently demonstrated the neutral fat-reducing effect ${ }^{23,24)}$, inhibitory effects on fat accumulation ${ }^{25}$ /body fat accumulation ${ }^{26}$, and the catabolic action ${ }^{27}$ of polyphenols with respect to lipid metabolism, suggesting that polyphenols reduce visceral fat.

It has been shown from previous basic studies that apple/hop bract-derived polyphenols containing procyanidins as the main component ${ }^{28,29)}$, especially apple polyphenols, inhibit an increase in serum cholesterol level ${ }^{30,31)}$, pro- $^{-}$ mote the activity of a fat-burning enzyme in the liver, acyl CoA oxidation enzyme, and reduce the activity of fatty acid synthase ${ }^{32}$, as reported for tea catechins. Twelve-week studies on apple/hop bract ingestion ${ }^{33-36)}$, mutagenicity tests, and acute/subacute toxicity studies have confirmed the safety of apple/hop bract polyphenols ${ }^{37,38}$. An apple contains approximately $200 \mathrm{mg}$ of polyphenols. In the United States, an apple polyphenol (Applephenon ${ }^{\circledR}$ ) was approved as a Generally Recognized as Safe (GRAS) product by the Food and Drug Administration (FDA). Considering that methods for extracting and processing these polyphenols have been established, they can be applied as functional food materials that reduce the serum cholesterol level, preventing obesity. The Japanese Society of Obesity defined Japanese patients with a body mass index (BMI) of 25 or more as being obese ${ }^{39}$.

We examined the usefulness of 12 -week ingestion of polyphenols (consisting mainly of procyanidins) in the pri- mary prevention of metabolic syndrome in obese patients with a BMI (obesity index) ranging from 23 to 30 .

\section{EXPERIMENTAL}

\subsection{Subjects}

From a group of male and female adult volunteers, we selected 74 subjects with a BMI ranging from 23 to 30 based on a preliminary medical assessment four weeks prior to ingestion. The subjects lived in Osaka area, Japan and engaged in normal works including company employees, civil officers, and homemakers. We excluded persons taking agents or supplements that may influence lipid metabolism, those with a history of food allergy, those in whom more than $200 \mathrm{ml}$ of blood had been collected (blood donation) within one month before the start of this study, or more than $400 \mathrm{ml}$ of blood had been collected within three months, and those who were considered to be ineligible by attending physicians. A physician who did not directly participate in this study assigned the 74 subjects to three groups based on the results of the preliminary medical assessment (hematology, physical examination) and computed tomography (CT) findings before administration began so that there were no marked differences in age, body weight, height, obesity index, blood pressure, neutral fat, total fat area (TFA), visceral fat area (VFA), subcuta-

Table 1 Demographic Data and Baseline Characteristics ${ }^{1}$.

\begin{tabular}{lccc}
\hline Parameters & Group A (Hop bract) & Group B (Apple) & Group C (Placebo) \\
\hline $\begin{array}{l}\text { Number } \\
\text { Male:Female Ratio }\end{array}$ & Male 15 Female 8 & Male 15 Female 9 & Male 15 Female 9 \\
\hline Age & $47.0 \pm 9.3$ & $47.8 \pm 10.8$ & $46.5 \pm 10.8$ \\
\hline Height (cm) & $164.7 \pm 7.5$ & $164.5 \pm 10.3$ & $165.3 \pm 8.4$ \\
\hline Body weight $(\mathrm{kg})$ & $70.0 \pm 9.1$ & $70.4 \pm 11.3$ & $70.9 \pm 8.8$ \\
\hline BM I & $25.7 \pm 2.0$ & $25.9 \pm 1.9$ & $25.9 \pm 2.2$ \\
\hline Waist $(\mathrm{cm})$ & $84.5 \pm 7.0$ & $86.4 \pm 7.3$ & $84.2 \pm 6.5$ \\
\hline Hip $(\mathrm{cm})$ & $98.7 \pm 4.6$ & $98.8 \pm 5.3$ & $99.9 \pm 4.1$ \\
\hline Waist/Hip Ratio & $0.86 \pm 0.06$ & $0.87 \pm 0.04$ & $0.84 \pm 0.05$ \\
\hline Systolic blood pressure (mmHg) & $127.5 \pm 15.1$ & $127.4 \pm 12.6$ & $124.6 \pm 15.8$ \\
\hline Diastolic blood pressure (mmHg) & $80.8 \pm 9.0$ & $77.0 \pm 9.3$ & $78.7 \pm 10.4$ \\
\hline Pulse (min) & $71.6 \pm 6.3$ & $69.3 \pm 7.0$ & $68.8 \pm 8.6$ \\
\hline Total cholesterol (mg/dl) & $218.7 \pm 37.0$ & $219.7 \pm 30.7$ & $221.8 \pm 28.9$ \\
\hline LDL-cholesteroa (mg/dl) & $140.2 \pm 33.6$ & $140.3 \pm 24.0$ & $142.7 \pm 26.2$ \\
\hline HDL-cholesteroal (mg/dl) & $52.9 \pm 13.4$ & $52.2 \pm 10.9$ & $52.3 \pm 11.5$ \\
\hline
\end{tabular}

${ }^{1}$ Each value is the mean $\pm \mathrm{SD}$. None of these characteristics were different among the groups at study start (Bonferroni's multiple comparison test). 
neous fat area (SFA), or waist/hip ratio. As shown in Table 1 , there were no significant differences in any parameters among the three groups.

We excluded three subjects (1 male in each group) that had a rapid increase in the neutral fat level 16 weeks after the start of this study (12 weeks after the start of ingestion) due to poor diet control, since the Dropout Review Committee confirmed that these dropouts were not associated with the test diets. Finally, we evaluated 71 subjects (45 males, 26 females).

This study was approved by the Soiken Clinical Trial Ethics Committee. The study contents and methods were explained to the subjects in accordance with the Helsinki declaration and written informed consent was obtained from all subjects.

\subsection{Test products}

As test dietary supplements, we used two kinds of capsules containing hop bract or apple polyphenols (150 $\mathrm{mg} /$ capsule) and a control polyphenol-free capsule (placebo capsule). To investigate the effects of polyphenols, the placebo capsule consisted of the same components excluding polyphenols (crystalline cellulose, tricalcium phosphate, sucrose fatty acid ester, glycerine fatty acid ester, fine silica dioxide). Prior to this study, attending physicians checked the three kinds of capsules and confirmed that there were no differences in taste, flavor, or packaging between the test and placebo capsules.

\subsection{Ingestion methods and schedule}

We conducted this randomized double-blind placebo-controlled comparative study as described below. A 20-week study period was established: 4-week observation, 12-week ingestion, and 4-week follow-up. The subjects were divided into three groups and the test capsules were administered as follows:

Group A: Ingestion of a hop bract polyphenol-containing capsule (150 mg/capsule). The subjects took four capsules before dinner (total: four capsules/day).

Group B: Ingestion of an apple polyphenol-containing capsule (150 mg/capsule). The subjects took four capsules before dinner (total: four capsules/day).

Group C: Ingestion of a placebo capsule $\left(150 \mathrm{mg} / \mathrm{cap}^{-}\right.$ sule). The subjects took four capsules before dinner (total: four capsules/day).

The subjects were instructed to maintain their normal daily life including diet, smoking, and exercise.

\subsection{Examination items}

All subject examinations were conducted or supervised by a physician.

\subsubsection{Blood tests}

The blood samples were collected from the brachial vein, processed, and analyzed in accordance with the relevant regulations. Prior to all examinations, the subjects were fasted for 12 hours or more to avoid the influence of extrinsic factors such as diet and exercise. After they were rested for 10 minutes or more following arrival, blood was collected in the sitting position.

In a preliminary medical assessment prior to the start of this study, we measured the levels of triglycerides, total cholesterol, LDL-cholesterol, HDL-cholesterol, free fatty acids, phospholipids, creatinine, $\mathrm{HbA}_{1 \mathrm{C}}$, GOT (AST), GPT (ALT), g-GTP, and fasting blood sugar.

In this study, hematology was performed five times, that is, at the start of ingestion, after 4,8 , and 12 weeks of ingestion, and four weeks after the end of ingestion (at the end of the four-week follow-up period). The parameters included triglycerides, total cholesterol, LDL-cholesterol, HDL-cholesterol, phospholipids, and non-esterified fatty acids (NEFA).

In addition, we measured adiponectin, PAI-1, leptin, hsCRP, GOT (AST), alkaline phosphatase (ALP), albumin, creatinine, $\mathrm{Na}, \mathrm{Ca}, \mathrm{Cl}, \mathrm{K}$, GPT (ALT), lactic acid dehydrogenase (LDH), total bilirubin, fasting blood sugar, insulin, unsaturated iron binding capacity (UIBC), Fe, g-GTP, total protein, urea nitrogen, $\mathrm{HbA}_{1 \mathrm{C}}$, and blood cell components (leukocytes, erythrocytes, hemoglobin level, hematocrit value, mean corpuscular volume (MCV), mean corpuscular hemoglobin $(\mathrm{MCH})$, mean corpuscular hemoglobin concentration (MCHC), and platelet count) at the start of ingestion and after 12 weeks of ingestion (at the end of ingestion).

2.4.2 CT

In the subjects, abdominal fat analysis by CT scan was performed. Using PC software for measuring visceral fat (Fat Scan, N2 System Co., Ltd.), we calculated TFA, VFA, and SFA from transverse L4/L5 intervertebral disk sections on abdominal CT.

The analysis was performed at the start of ingestion and after 12 weeks of ingestion (at the end of ingestion). As a rule, it was performed within four days before and after examination. On the day of examination, it was performed after a 4-hour fast (water was restricted for two hours prior to examination).

\subsubsection{Physical examination}

In the preliminary medical examination prior to the start of this study, we measured height, body weight, waist/hip ratio, blood pressure, and pulse rate. The obesity index (BMI) was calculated from the height and body weight.

In this study, body weight, waist/hip ratio, blood pressure, and pulse rate were measured six times, that is, four weeks before the start of ingestion (observation period), at the start of ingestion, after 4, 8, and 12 weeks of ingestion, and four weeks after the end of ingestion (at the end of the four-week follow-up period). BMI was calculated from the height obtained in the preliminary test. After the subjects were rested for 10 minutes or more following arrival, blood pressure and pulse rate were measured from the left 
brachium in the sitting position while clothed by skilled nurses. Body weight was measured using a TBF-614 meter (Tanita).

2.4.4 Diet survey and measurement of exercise

The subjects were instructed to use a diary to record their dietary intake and the intensity of exercise (walking) for three days before each examination at the start of ingestion, after 4,8 , and 12 weeks of ingestion, and four weeks after the end of ingestion (at the end of the fourweek follow-up period) as well as after 2, 6, and 10 weeks of ingestion during the ingestion period.

We analyzed the data over three days before each examination at the start of ingestion, after 4,8 , and 12 weeks of ingestion, and four weeks after the end of ingestion (at the end of the four-week follow-up period). In the dietary record analysis, dieticians calculated nutrient intake (energy, protein, fat, carbohydrate, cholesterol, and dietary fiber). For exercise, the subjects were instructed to record daily walking activity using a pedometer. They recorded alcohol intake in the diary every day during the study period.

\subsubsection{Physician interviews of subjects}

At the time of the preliminary medical examination and during this study, physicians interviewed subjects six times, that is, four weeks before the start of ingestion (observation period), at the start of ingestion, after 4,8 , and 12 weeks of ingestion, and four weeks after the end of ingestion (at the end of the four-week follow-up period), to ascertain the incidence of symptoms such as general malaise, anorexia, nausea, diarrhea, vomiting, and headache as well as other adverse reactions.

\subsubsection{Statistical analysis}

All values were expressed as the mean \pm standard deviation. Concerning the physical examination findings and the pattern of changes in serum lipids during the ingestion period, we analyzed the alternate actions of the test capsules with the ingestion period between Groups $\mathrm{A}$ and $\mathrm{C}$ and between Groups B and C by two-way repeated measures analysis of variance (ANOVA). Furthermore, Bonferroni's multiple comparison test was used to compare the hematological data, physical examination findings, CT findings, nutrient intake, and exercise between Groups A and B. In each test capsule group, the data were compared with the value at the start of ingestion using Bonferroni's multiple test or the paired t-test. We employed SPSS Ver. 11.5 statistical software (SPSS Co., Ltd.). In all tests, $P<0.05$ was regarded as significant (paired test).

\section{RESULTS}

\subsection{Serum lipid levels}

The changes in the total cholesterol level are shown in Fig. 1. The total cholesterol level in Group B was signifi- cantly decreased $(P<0.05)$ compared with Group C after four weeks of ingestion and compared with the baseline after 12 weeks. This trend was also noted in Group A. Blood samples were further separated into various lipoprotein fractions as presented in Table 2. A lipoprotein lipid fraction test showed a significant decrease in the LDLcholesterol level in Group B $(P<0.05)$ compared with the baseline after 8 and 12 weeks of ingestion. There was a significant decrease in the LDL-cholesterol level in Group A $(P<0.05)$ compared with Group $\mathrm{C}$ after four weeks.

\subsection{Changes in the abdominal fat area}

The changes in TFA, VFA, and SFA are shown in Fig. 2.

We measured the abdominal fat area and there was a slight decrease in VFA in Group B. TFA was slightly increased in Group C, higher than the values in Groups A and B. In Groups A and B, TFA, VFA, and SFA were lower than the values in Group $C$, suggesting the visceral fatreducing effects of the apple/hop bract polyphenol-containing capsules.

\subsection{Physical examination}

The changes in body weight in individual subjects were investigated as shown in Fig. 3. In Groups A and B, these parameters serially decreased after four weeks of ingestion, although there were no significant differences compared with baseline values. The changes in body weight and BMI differed between Groups B and C. There were no

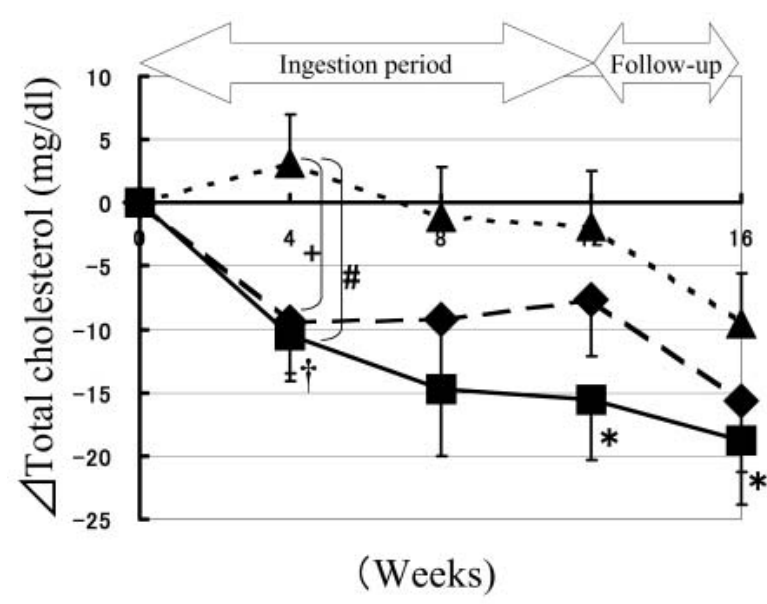

Fig. 1 Changes in Serum Yotal Cholesterol Levels by 12week Ingestion of Polyphenols in Group A (Hop bract) $(\bullet)$, Group B (Apple) $(\boldsymbol{\square})$, and Group C (Placebo) ( $\mathbf{\Delta})$.

Values are mean $\pm \mathrm{SE}$. Symbols indicate differences among groups, $+: P<0.1, \#: P<0.05$. Symbols indicate differences versus baseline, $\dagger: P<0.1, *$ : $P<0.05$. Comparisons were undertaken using Bonferroni's multiple comparison test. 


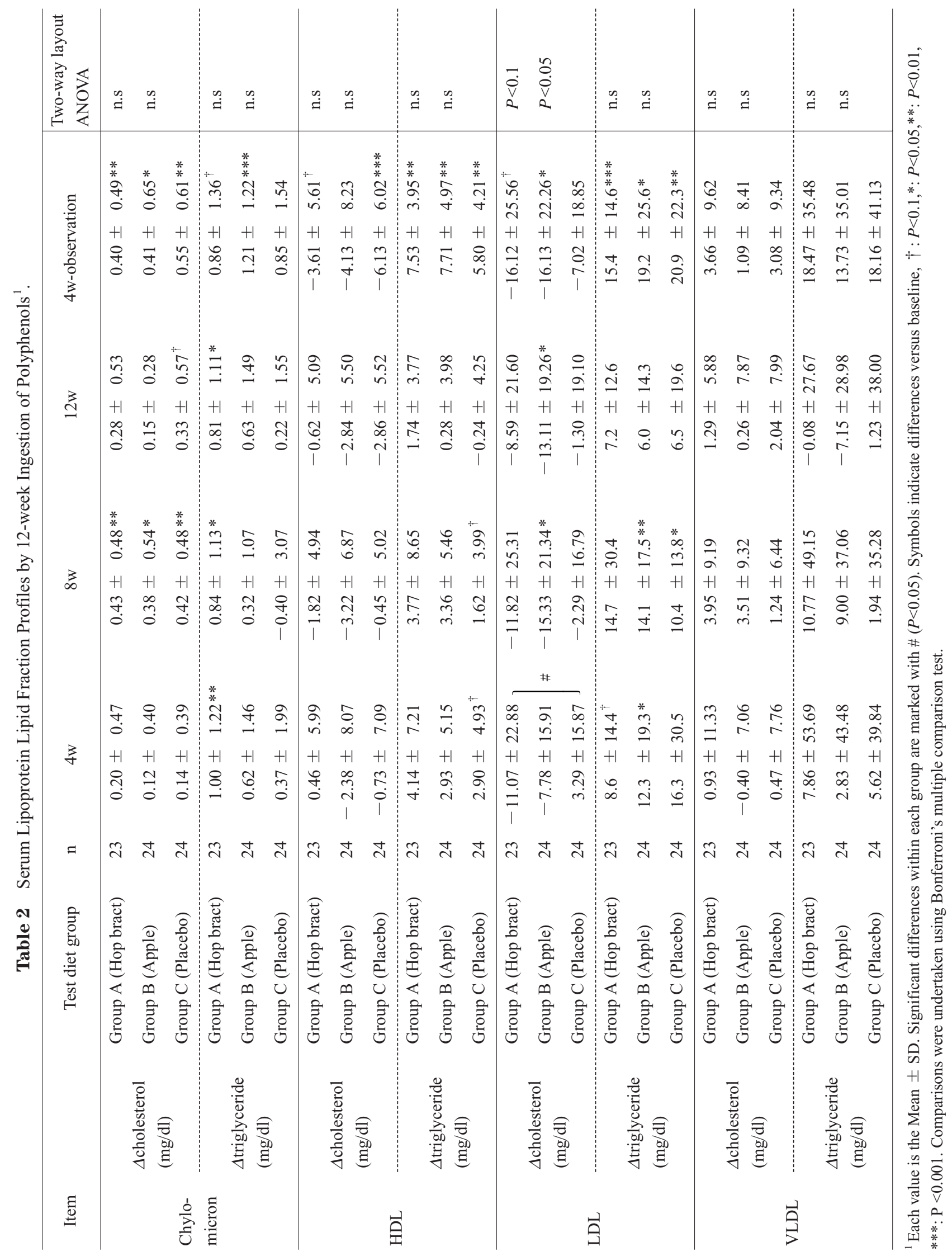


(A)

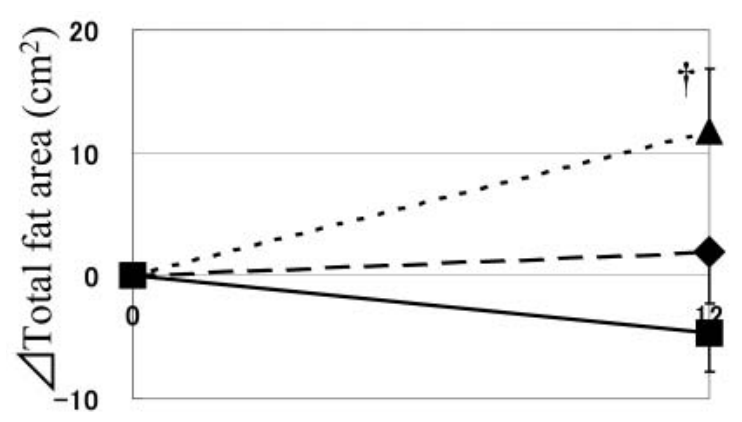

(Weeks)

(B)

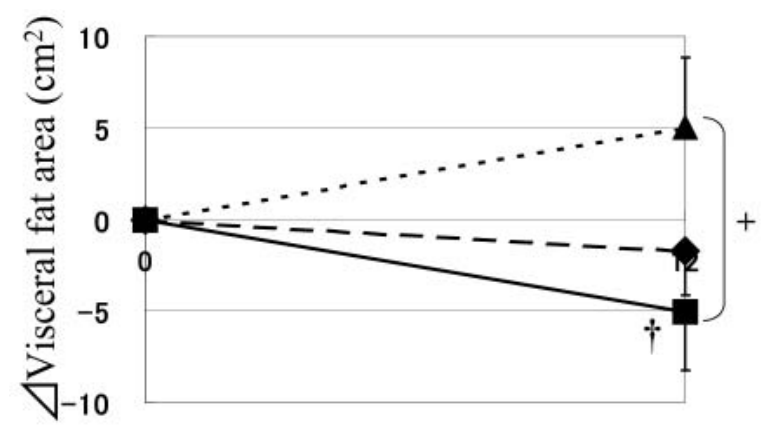

(Weeks)

(C)

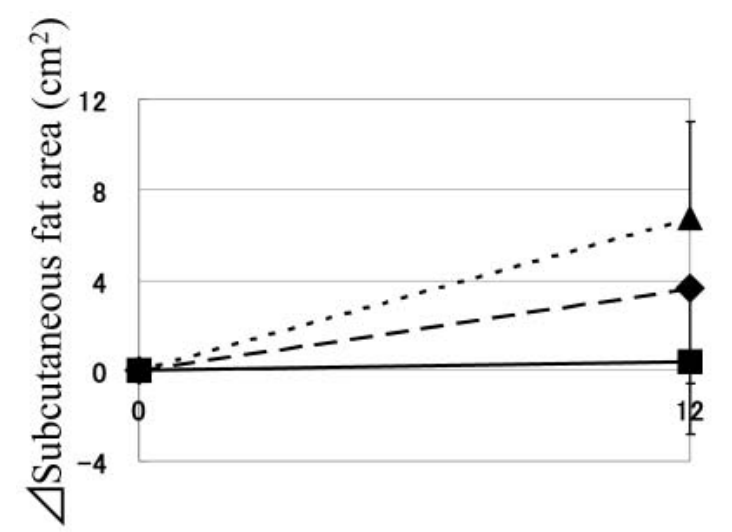

(Weeks)

Fig. 2 Changes in the Abdominal Fat Areas by 12-week Ingestion of Polyphenols in Group A (Hop bract) $(\diamond)$, Group B (Apple) (ם), and Group C (Placebo) (А).

(A) Total fat area. (B) Visceral fat area. (C) Subcutaneous fat area. Values are mean \pm SE. Symbols indicate differences among groups, +: $P<0.1$. Symbols indicate differences versus baseline, $\dagger: P<0.1$. Comparisons were undertaken using Bonferroni's multiple comparison test.

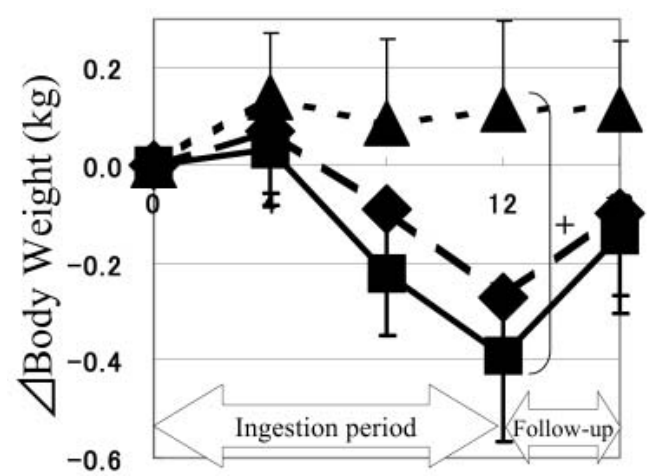

\section{(Weeks)}

Fig. 3 Changes in Body Weight by 12-week Ingestion of Polyphenols in Group A (Hop bract) ( ), Group B

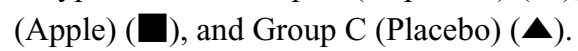

Values are mean \pm SE. Symbols indicate differences among groups, $+: P<0.1$. Comparisons were undertaken using Bonferroni's multiple comparison test.

abnormalities in blood pressure or pulse (data not shown).

\subsection{Adipocytokines}

The adiponectin test results are shown in Table 3. In Group C, the adiponectin level in blood decreased significantly after 12 weeks of ingestion $(P<0.001)$ compared with the baseline. There were significant differences between Groups A and C $(P<0.05)$ and between Groups B and C $(P<0.01)$. No significant differences were observed in leptin or PAI-1 concentrations.

\subsection{Nutrient intake, alcohol intake, and exercise}

There was a significant difference in fat intake level after four weeks of ingestion between Groups A and B $(P<0.05)$. However, there were no significant differences in calorie, protein, carbohydrate, cholesterol, dietary fiber, and alcohol intakes or exercise intensity. During the ingestion period, there were no significant changes in the parameters other than fat intake level in any group (Table 4).

\subsection{Changes in the biochemical and hematological data}

In Group A, the values of creatinine $(0.94 \pm 0.18 \rightarrow 1.03$ $\pm 0.16 \mathrm{mg} / \mathrm{dl}), \mathrm{Cl}(105.1 \pm 2.3 \rightarrow 108.0 \pm 2.0 \mathrm{mEq} / \mathrm{l}), \mathrm{UIBC}$ $(236.2 \pm 80.9 \rightarrow 252.7 \pm 71.5 \mu \mathrm{g} / \mathrm{dl}), \mathrm{Na}(144.3 \pm 2.2 \rightarrow$ $147.6 \pm 1.6 \mathrm{mEq} / \mathrm{l})$, albumin $(4.53 \pm 0.18 \rightarrow 4.65 \pm 0.19$ $\mathrm{g} / \mathrm{dl}), \mathrm{MCH}(30.8 \pm 1.8 \rightarrow 30.9 \pm 1.8 \mathrm{pg})$, and $\mathrm{MCHC}(33.5$ $\pm 1.0 \rightarrow 33.8 \pm 1.0 \%$ ) were significantly increased. The levels of LDH $(200.7 \pm 30.0 \rightarrow 193.1 \pm 34.1 \mathrm{IU} / \mathrm{l})$, MCV $(91.8 \pm 4.0 \rightarrow 91.3 \pm 3.9 \mathrm{fl})$, and potassium $(4.20 \pm 0.32 \rightarrow$ $4.03 \pm 0.25 \mathrm{mEq} / \mathrm{l})$ were significantly decreased. 
Table 3 Changes in Adipocytokine Concentrations in Blood by 12-week Ingestion of Polyphenols ${ }^{1}$.

\begin{tabular}{|c|c|c|c|c|c|c|c|}
\hline Item & Standard value & Test diet group & $\mathrm{n}$ & Baseline & $12 \mathrm{w}$ & $\Delta$ & \\
\hline Adiponectin & $3.8-16.6$ & Group A (Hop bract) & 23 & $6.67 \pm 2.95$ & $6.49 \pm 2.93$ & $-0.17 \pm 0.81$ & \\
\hline \multirow[t]{2}{*}{$(\mu \mathrm{g} / \mathrm{ml})$} & & Group B (Apple) & 24 & $6.01 \pm 2.35$ & $6.05 \pm 2.70$ & $0.04 \pm 0.87$ & \# \\
\hline & & Group C (Placebo) & 24 & $7.10 \pm 2.97$ & $6.48 \pm 2.46^{* * *}$ & $\left.-0.84 \pm 0.75^{* * *}\right]$ & \\
\hline PAI-1 & $0-50$ & Group A (Hop bract) & 23 & $51.5 \pm 29.3$ & $48.0 \pm 16.3$ & $-3.6 \pm 28.3$ & \\
\hline \multirow[t]{2}{*}{$(\mathrm{mg} / \mathrm{dl})$} & & Group B (Apple) & 24 & $52.1 \pm 29.6$ & $46.8 \pm 19.7$ & $-5.3 \pm 21.1$ & \\
\hline & & Group C (Placebo) & 24 & $43.7 \pm 18.9$ & $40.6 \pm 16.8$ & $-3.1 \pm 17.6$ & \\
\hline Leptin & & Group A (Hop bract) & 23 & $8.00 \pm 5.45$ & $8.57 \pm 6.35$ & $0.57 \pm 2.45$ & \\
\hline \multirow[t]{2}{*}{$(\mathrm{ng} / \mathrm{ml})$} & & Group B (Apple) & 24 & $8.25 \pm 4.81$ & $8.25 \pm 4.77$ & $0.00 \pm 1.72$ & \\
\hline & & Group C (Placebo) & 24 & $8.08 \pm 5.07$ & $8.50 \pm 4.58$ & $0.42 \pm 2.90$ & \\
\hline
\end{tabular}

${ }^{1}$ Each value is the mean $\pm \mathrm{SD}$. Symbols indicate differences among groups, \#: $P<0.05$, \#\#: $P<0.01$. Symbols indicate differences versus baseline, ${ }^{* *}: P<0.001$. Comparisons were undertaken using Bonferroni's multiple comparison test.

In Group B, the values of creatinine, $\mathrm{Cl}(105.1 \pm 1.8 \rightarrow$ $107.7 \pm 1.7 \mathrm{mEq} / \mathrm{l}), \mathrm{Na}(144.7 \pm 2.1 \rightarrow 147.3 \pm 1.9 \mathrm{mEq} / \mathrm{l})$, albumin $(4.55 \pm 0.16 \rightarrow 4.64 \pm 0.17 \mathrm{~g} / \mathrm{dl})$, and $\mathrm{MCHC}(33.4$ $\pm 0.9 \rightarrow 33.8 \pm 0.9 \%)$ were significantly increased. The hematocrit value $(44.8 \pm 3.5 \rightarrow 43.7 \pm 3.5 \mathrm{~g} / \mathrm{dl}), \mathrm{MCV}(92.1$ $\pm 3.3 \rightarrow 90.8 \pm 3.9 \mathrm{fl})$, and platelet count $(25.9 \pm 6.5 \rightarrow$ $25.2 \pm 6.6 \times 104 / \mu \mathrm{l})$ were significantly decreased.

In Group C, the values of BUN $(14.9 \pm 3.3 \rightarrow 16.0 \pm 3.9$ $\mathrm{mg} / \mathrm{dl})$, creatinine $(0.97 \pm 0.18 \rightarrow 1.06 \pm 0.21 \mathrm{mg} / \mathrm{dl})$, total bilirubin $(0.69 \pm 0.28 \rightarrow 0.81 \pm 0.34 \mathrm{mg} / \mathrm{dl}), \mathrm{Cl}(105.8 \pm 2.1$ $\rightarrow 108.4 \pm 2.2 \mathrm{mEq} / \mathrm{l}), \mathrm{Na}(145.2 \pm 1.9 \rightarrow 147.8 \pm 1.7$ $\mathrm{mEq} / \mathrm{l})$, albumin $(4.41 \pm 0.18 \rightarrow 4.56 \pm 0.26 \mathrm{~g} / \mathrm{dl})$, and MCHC (33.3 $\pm 0.5 \rightarrow 33.7 \pm 0.6 \%)$ were significantly increased. MCV $(91.1 \pm 3.6 \rightarrow 90.5 \pm 3.6 \mathrm{fl})$ was significantly decreased.

These changes were within the reference ranges, and were not considered to be significant.

\subsection{Adverse reactions}

During the ingestion period, cold symptoms were noted in 11 patients, diarrhea or loose stools in five, and other digestive symptoms (anorexia) in two. However, ingestion was continued based on the judgment of the attending physicians and most symptoms spontaneously resolved during the study period.

\section{DISCUSSION}

We examined the benefits of 12 -week continuous ingestion of apple/hop bract polyphenol-containing capsules in the primary prevention of metabolic syndrome in male and female adults with a BMI ranging from 23 to 30 and who were concerned about an increase in their visceral fat.

The total cholesterol and LDL-cholesterol levels were significantly decreased in Group B. There was a tendency towards decrease in Group A also. Concerning the mechanism by which polyphenols decrease the cholesterol level, it is possible that polyphenols inhibit the enterohepatic circulation of cholesterol and bile acid by binding to cholesterol and/or bile acid and enhancing fecal excretion. Enhanced liver cholesterol isomerization to bile acid may facilitate LDL particle uptake into blood, decreasing the number of serum cholesterol-activating LDL receptors in the liver. Micelle formation is known to boost lipid molecule absorption. Cholesterol biosynthesis rate-determining enzyme (HMG-CoA reductase) activity in the liver was reduced in rats fed apple polyphenol compared to rats that were not ${ }^{30)}$. The cholesterol-reducing effects of polyphenols in humans could be reproduced in our present study.

It is assumed that the mechanism by which proanthocyanidins decrease the visceral fat level is as follows: proanthocyanidins, that is, condensed tannins, have been reported to reduce dietary efficiency in livestock and a vegetable-derived component with the above action has been investigated ${ }^{40-42)}$. There is also evidence that condensed tannins exhibit physiological actions such as antioxidant activity and inhibition of lipase, thereby preventing obesity ${ }^{43}$. When diets differing in terms of their tannin content were given to rats, body weight significantly decreased with an increase in the tannin content ${ }^{40}$. In chickens, a high-tannin diet decreased the rate of weight gain and dietary efficiency ${ }^{41,44}$. In pigs, a high-tannin diet significantly increased dietary intake, but decreased dietary efficiency, although there was no significant decrease in body weight. In addition, it significantly decreased the rate of digestion and total energy ${ }^{42}$. Furthermore, after tannins were extracted and removed from a high-tannin diet, the diet was given to rats. Body weight 


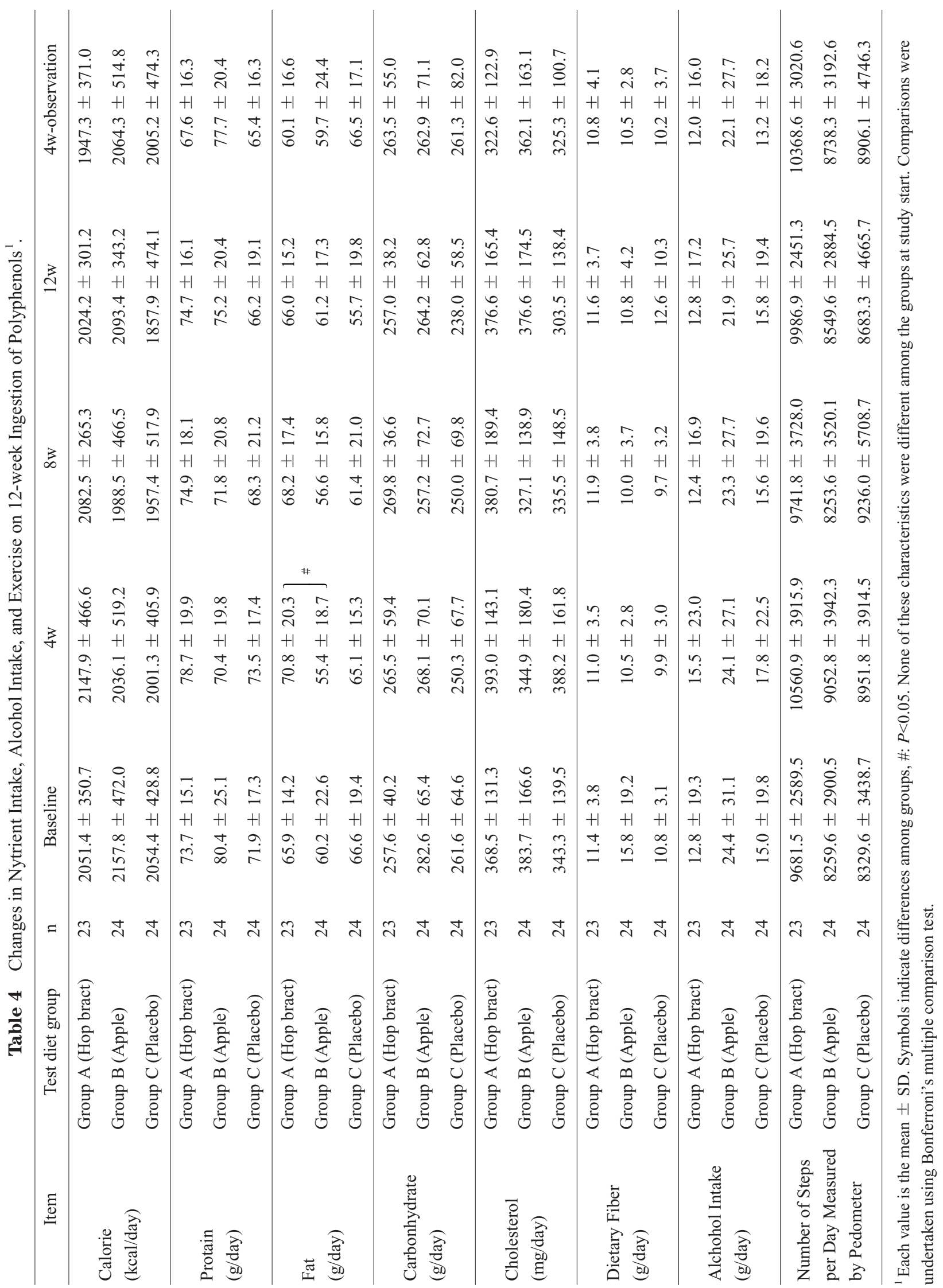


was significantly lower than it was in rats fed a diet from which the tannin had been extracted ${ }^{44}$. A high-tannin sorghum diet given to rabbits significantly decreased the rate of weight gain and dietary efficiency. The activities of $\alpha$-amylase, trypsin, and lipase in the upper small intestinal contents were inhibited in proportion to the tannin-containing dietary intake ${ }^{45}$. In Zucker obese rats, tannins inhibited fat-metabolizing enzymes such as pancreatic lipase, lipoprotein lipase, and glycerophosphate dehydrogenase, and increased lipid breakdown, preventing obesity ${ }^{18}$. To investigate the influence of apple-derived polyphenols on digestion/absorption, we conducted a study using a starch-digesting enzyme extracted from rat small intestine and pig pancreatic lipase. Apple-derived polyphenols markedly reduced the activities of these digestive enzymes. On starch and lipid load tests using mice, these polyphenols also inhibited absorption. In addition, continuous ingestion decreased the fasting neutral fat level ${ }^{46}$. When a high-fat diet was given to rats, apple-derived polyphenols, mainly consisting of procyanidins, significantly decreased the visceral fat level and significantly increased the fecal free fatty acid level ${ }^{47}$.

Animal experiments have suggested that the mechanism by which apple-derived polyphenols decrease the visceral fat level involves inhibition of lipid ${ }^{48,49)}$ or glucose ${ }^{50)}$ absorption in the digestive tract, as described above. The results of this human study also support the contention that inhibition of lipid absorption in the intestinal tract by apple/hop bract polyphenols is involved. However, it has also been reported that catechins decrease body fat without inhibiting lipid absorption ${ }^{51}$. Therefore, mechanisms other than inhibition of lipid absorption may play a role. Recently, many animal and human studies have reported that catechins promote lipid catabolism ${ }^{27,52,53)}$. This suggests the enhancement of lipid metabolism is related to an increase in oxygen consumption and a decrease in the respiratory quotient. The results of this study also suggest the involvement of polyphenols in visceral fat reduction. A high-fat diet containing apple-derived polyphenols mainly consisting of procyanidins (condensed tannins) given to rats significantly decreased the visceral fat level compared to a polyphenol-free high-fat diet. In particular, it markedly reduced the mesenteric fat level. In the liver, it enhanced beta oxidation and reduced fatty acid synthase activity, inhibiting the expression of fatty acid-synthesizing genes in the liver ${ }^{32}$. According to another study, when applederived polyphenols were given to rats that had been acclimated to a standard diet, they inhibited the expression of fatty acid-synthesizing genes in the liver. The visceral fat level in the group fed a standard diet containing polyphenols was significantly lower than that in the polyphenolfree diet group, although there was no marked difference in body weight ${ }^{54}$. These studies suggest that polyphenols enhanced lipid metabolism.
The reason why the reduction in the visceral fat level was not large may be associated with the dose. One study on polyphenols in humans indicated that ingestion of red wine $(0.8 \mathrm{~g}$ alcohol $/ \mathrm{kg} /$ day $)$ containing high-level polyphenols (mainly consisting of procyanidins) for 14 days significantly inhibited oxidation of low-density lipoproteins in blood, demonstrating the French paradox (a high-fat diet and a low incidence of heart disease ${ }^{55}$. This reflects the fact that when the alcohol content of red wine is $13 \%$, human body weight is $50 \mathrm{~kg}$ and polyphenol content is approximately $0.2 \%$, the polyphenol level is calculated as approximately $616 \mathrm{mg}$. When dietary intake in animals ranges from 70 to $150 \mathrm{~g} /$ day $/ \mathrm{kg}$ with a 60 -fold intraspecies safety factor between animals and humans, a 1\% addition of polyphenols to a diet corresponds to 580 to $1,250 \mathrm{mg} /$ day in humans. Considering dietary intake in humans, the optimal intake of apple-derived polyphenols mainly consisting of procyanidins is probably in the vicinity of $600 \mathrm{mg} /$ day. For the above reasons, polyphenols at approximately 600 $\mathrm{mg} /$ day may be effective and an additional large-scale longterm study should be conducted to confirm this.

Nagao et al. also reported that there were no significant differences in the leptin level in a study involving 20-week ingestion of catechins ${ }^{56)}$. PAI-1 is a fibrinolytic factor involved in the inflammatory response and is known to promote blood coagulation. It is also known that an increase in the PAI-1 level promotes arteriosclerosis and that there is a correlation between PAI- 1 and VFA ${ }^{10)}$. In the present study, Groups A and B showed a slight decrease in the PAI-1 level, although there were no significant differences. Adiponectin is a hormone secreted by adipose cells, as reported for leptin. Many studies have indicated its relationship to insulin resistance ${ }^{11-13)}$. It is known that the adiponectin level decreases with an increase in visceral fat. In this study, there were significant differences between Group C and Groups A/B after 12 weeks of ingestion. In Group C, the visceral fat level increased and the adiponectin level significantly decreased. However, in Groups A and B, especially in Group B, the visceral fat level reduced, maintaining high levels of adiponectin.

Concerning the safety of the test capsules used in this study, the hematological data other than serum lipids after 12 -week ingestion of the test capsules $(600 \mathrm{mg} /$ day) did not show any abnormalities in liver/kidney function or electrolytes. Catechins are known to inhibit intestinal absorption via iron binding ${ }^{43,58)}$. However, in this study, neither our analysis involving all subjects nor stratified analysis with respect to gender showed any significant changes in the serum Fe level during the ingestion period. Among the subjects, UIBC, which reflects the iron binding reserve, increased in Group A. However, the stratified analysis did not show any significant differences. In addition, concerning adverse reactions, no subject showed anemia, but cold symptoms, diarrhea/loose stools, and other digestive 


\section{Y. Nagasako-Akazome, T. Kanda, Y. Ohtake et al.}

symptoms (anorexia) were observed in 11, five, and two subjects during the study period, respectively. Most symptoms were transient and all resolved spontaneously during the study period (one patient with loose stools is being followed up). In the placebo capsule group, similar symptoms were also noted; therefore, the above symptoms may not have been associated with the test capsules. These findings suggest that 12 -week ingestion of the test capsules is safe.

\section{CONCLUSION}

We performed a randomized double-blind, placebo-controlled study of normal weight or moderately obese male and female subjects (71 subjects) with a body mass index ranging from 23 to 30 to evaluate the efficacy of polyphenols extracted from apples and hop bract (600 mg/day).

(1) Polyphenol-containing capsules decreased total cholesterol and LDL-cholesterol levels significantly. The effects of the apple polyphenol-containing capsules were more marked than those of the hop bract polyphenol-containing capsules.

(2) The visceral fat area and the level of adiponectin in the apple polyphenol group improved as compared with the control group.

(3) There were no clinical problems in the blood and physical examinations, and no adverse reactions attributable to the study substance were observed during the ingestion period.

12-week ingestion of apple/hop bract polyphenols was safe in this study, suggesting that these food materials are useful for preventing lifestyle-related diseases in the community in which obesity is an important problem.

\section{References}

1. World Health Organization. Preventing chronic diseases: a vital investment. World Health Organization, Geneva, Schweiz (2005).

2. Yach, D.; Stuckler, D.; Brownell, K.D. Epidemiologic and economic consequences of the global epidemics of obesity and diabetes. Nat. Med. 12, 62-66 (2006).

3. Nelson, L.H.; Tucker, L.A. Diet composition related to body fat in a multivariate study of 203 men. J. Am. Diet. Assoc. 96, 771-777 (1996).

4. Kobayashi, H.; Nakamura, T.; Miyaoka, K.; Nishida, M.; Funahashi, T.; Yamashita, S.; Matsuzawa, Y. Visceral fat accumulation contributes to insulin resistance, small-sized low-density lipoprotein, and progression of coronary artery disease in middle-aged non-obese Japanese men. Jpn. Circ. J. 65, 193-199 (2001).

5. Fujioka, S.; Matsuzawa, Y.; Tokunaga, K.; Tarui, S. Con- tribution of intra-abdominal fat accumulation to the impairment of glucose and lipid metabolism in human obesity. Metab. Clin. Exp. 36, 54-59 (1987).

6. Mapfei, M.; Halaas, J.; Ravussin, E.; Pratley, R.E.; Lee, G.H.; Zhangs, Y.; Fei, H.; Kim, S.; Lallone, R.; Ranganathan, S.; Kern, P.A.; Friedman, J.M. Leptin levels in human and rodent: Measurement of plasma leptin and ob RNA in obese and weight-reduced subjects. Nat. Med. 1, 1155-1161 (1995).

7. Hotamisligil, G.S.; Arner, P.; Caro, J.F.; Atkinson, R.L.; Spiegelman, B.M. Increased adipose tissue expression of tumor necrosis factor- $\alpha$ in human obesity and insulin resistance. J. Clin. Invest. 95, 2409-2415 (1995).

8. Shimomura, I.; Funahashi, T.; Takahashi, M.; Maeda, K.; Kotani, K.; Nakamura, T.; Yamashita, S.; Miura, M.; Fukuda, Y.; Takemura, K.; Tokunaga, K.; Matsuzawa, Y. Enhanced expression of PAI-1 in visceral fat: Possible contributor to vascular disease in obesity. Nat. Med. 2, 800-803 (1996).

9. Arita, Y.; Kihara,S.; Ouchi, N.; Takahashi, M.; Maeda, K.; Miyagawa, J.I.; Hotta, K.; Shimomura, I.; Nakamura, T.; Miyaoka, K.; Kuriyama, H.; Nishida, M.; Yamashita, S.; Okubo, K.; Matsubara, K.; Muraguchi, M.; Ohmoto, Y.; Funahashi, T.; Matsuzawa, Y. Paradoxical decrease of an adipose-specific protein, adiponectin, in obesity. Biochem. Biophys. Res. Commun. 257, 79-83 (1999).

10. Funahashi, T.; Nakamura, T.; Shimomura, I.; Maeda, K.; Kuriyama, H.; Takahashi, M.; Arita, Y.; Kihara, S.; Matsuzawa, Y. Role of adipocytokines on the pathogenesis of atherosclerosis in visceral obesity. Intern. Med. 38, 202-206 (1999).

11. Weyer, C.; Funahashi, T.; Tanaka, S.; Hotta, K.; Matsuzawa, Y.; Pratley, R.E.; Tataranni, P.A. Hypoadiponectinemia in obesity and type 2 diabetes: Close association with insulin resistance and hyperinsulinemia. J. Clin. Endocrinol. Metab. 86, 1930-1935 (2001).

12. Hotta, K.; Funahashi, T.; Bodkin, N.L.; Ortmeyer, H.K.; Arita, Y.; Hansen, B.C.; Matsuzawa, Y. Circulating concentrations of the adipocyte protein adiponectin are decreased in parallel with reduced insulin sensitivity during the progression to type 2 diabetes in rhesus monkeys. Diabetes. 50, 1126-1133 (2001).

13. Stefan, N.; Vozarova, B.; Funahashi, T.; Matsuzawa, Y.; Weyer, C.; Lindsay, R.S.; Youngren, J.F.; Havel, P.J.; Pratley, R.E.; Bogardus, C.; Tataranni, P.A. Plasma adiponectin concentration is associated with skeletal muscle insulin receptor tyrosine phosphorylation, and low plasma concentration precedes a decrease in whole-body insulin sensitivity in humans. Diabetes. 51, 1884-1888 (2002).

14. Ryo, M.; Nakamura, T.; Kihara, S.; Kumada, M.; Shibazaki, S.; Takahashi, M.; Nagai, M.; Matsuzawa, Y.; Funahashi, T. Adiponectin as a biomarker of the metabolic syndrome. Circulation J. 68, 975-981 (2004). 
15. Trujillo, M.E.; Scherer, P.E. Adiponectin-Journey from an adipocyte secretory protein to biomarker of the metabolic syndrome. J. Intern. Med. (GBR). 257, 167175 (2005).

16. Ushitani, K.; Amano, T.; Yoshibe, H. Effects of health foods. Characteristics and effects of tea polyphenol. New Food Ind. 41, 49-54 (1999).

17. Ina, K.; Sakata, K.; Nakamura, K. et al. in chemical composition and function of tea. (Ina, K.; Sakata, K.; Tomita, K.; Isemura, M. ed.). Kougaku Shuppan, Kanagawa, Japan, pp.6-26 (2002).

18. Yoshikawa, M.; Shimoda, H.; Nishida, N.; Takada, M.; Matsuda, H. Salacia reticulata and its polyphenolic constituents with lipase inhibitory and lipolytic activities have mild antiobesity effects in rats. J. Nutr. 132, 1819-1824 (2002).

19. Fujiki, H.; Suganuma, M.; Okabe, S.; Sueoka, N.; Komori, A.; Sueoka, E.; Kozu, T.; Tada, Y.; Suga, K.; Imai, K.; Nakachi, K. Cancer inhibition by green tea. Mutat. Res. Fundam. Mol. Mech. Mutagen. 402, 307310 (1998).

20. Valsa, A.K.; Sudheesh, S.; Vijayalakshmi, N.R. Effect of catechin on carbohydrate metabolism. Indian J. Biochem. Biophys. 34, 406-408 (1997).

21. Henry, J.P.; Stephens-Larson, P. Reduction of chronic psychosocial hypertension in mice by decaffeinated tea. Hypertension. 6, 437-444 (1984).

22. Kajimoto, O.; Kajimoto, Y.; Yabune, M.; Nozawa, A.; Nagata, K.; Kakuda, T. Tea catechins reduce serum cholesterol levels in mild and borderline hypercholesterolemia patients. J. Clin. Biochem. Nutr. 33, 101-111 (2003).

23. Chan, P.T.; Fong, W.P.; Cheung, Y.L.; Huang, Y.; Ho, W.K.K.; Chen, Z.Y. Jasmine green tea epicatechins are hypolipidemic in hamsters (Mesocricetus auratus) fed a high fat diet. J. Nutr. 129, 1094-1101 (1999).

24. Nanjo, F.; Hara, Y.; Kikuchi, Y. Effects of tea polyphenols on blood rheology in rats fed a high-fat diet. in Food Phytochemicals for Cancer Prevention II. Teas, Spices and Herbs. Anonymous American Chemical Society, Washington DC, USA, pp. 76-82 (1994).

25. Chaudhari, P.N.; Hatwalne, V.G. Effect of epicatechin on liver lipids of rats fed with choline deficient diet. Ind. J. Nutr. Diet. 14, 136-139 (1977).

26. Ishigaki, A.; Tonooka, F.; Matsumoto, N. Suppression of the accumulation of body and liver fat by tea catechin. 309-313 (1991).

27. Dulloo, A.G.; Seydoux, J.; Girardier, L.; Chantre, P.; Vandermander, J. Green tea and thermogenesis: Interactions between catechin-polyphenols, caffeine and sympathetic activity. Int. J. Obes. 24, 252-258 (2000).

28. Yanagida, A.; Shoji, T.; Shibusawa, Y. Separation of proanthocyanidins by degree of polymerization by means of size-exclusion chromatography and related techniques. J. Biochem. Biophys. Methods. 56, 311322 (2003).

29. Kurumatani, M.; Fujita, R.; Tagashira, M.; Shoji, T.; Kanda, T.; Ikeda, M.; Shoji, A.; Yanagida, A.; Shibusawa, Y.; Shindo, H.; Ito, Y. Analysis of polyphenols from hop bract region using CCC. J. Liq. Chromatogr. Relat. Technol. 28, 1971-1983 (2005).

30. Osada, K.; Suzuki, T.; Kawakami, Y.; Senda, M.; Kasai, A.; Sami, M.; Ohta, Y.; Kanda, T.; Ikeda, M. Dosedependent hypocholesterolemic actions of dietary apple polyphenol in rats fed cholesterol. Lipids. 41, 133-139 (2006).

31. Nagasako-Akazome, Y.; Kanda, T.; Ikeda, M.; Shimasaki, H. Serum cholesterol-lowering effect of apple polyphenols in healthy subjects. J. Oleo Sci. 54, 143151 (2005).

32. Osada, K.; Funayama, M.; Fuchi, S.; Sami, M.; Ohta, Y.; Kanda, T.; Ikeda, M. Effects of dietary procyanidins and tea polyphenols on adipose tissue mass and fatty acid metabolism in rats on a high fat diet. J. Oleo Sci. 55, 79-89 (2006).

33. National Food Research Institute, Apple. in Food Encyclopaedia (National Food Research Institute ed.) Asakura Publishing Co., Ltd., Tokyo, Japan, pp. 62-63 (2001).

34. Anonymous Apple, foodstuff in season and fruits of four seasons. (Kodansha ed.) Kodansha Ltd., Tokyo, Japan, pp. 64-68 (2001).

35. Blumenthal, M. Herbal medicine, expanded commission $E$ monographs in Integrative Medicine Communications, Newton. Anonymous pp. 193-195 (2000).

36. DeLyser, D.Y.; Kasper, W. J. Hopped Beer in The Case for Cultivation. Anonymous Econ. Botany, pp. 166-170 (1994).

37. Shoji, T.; Akazome, Y.; Kanda, T.; Ikeda, M. The toxicology and safety of apple polyphenol extract. Food Chem. Toxicol. 42, 959-967 (2004).

38. Akazome, Y.; Honma, D.; Tagashira, M.; Kanda, T.; Yasue, M.; Otake, Y. Safety evaluation of polyphenols extracted from hop bracts. Food Chem. Toxicol. (2007) in press.

39. Ministry of Health, Labour and Welfare, Health and Nutrition National Survey Report, 2003 (Health and Nutritional Information Workshop ed.) Dai-ichi Shuppan Publishing Co. Ltd., Tokyo, Japan, p.152 (2004).

40. Jambunathan, R.; Mertz, E.T. Relationship between tannin levels, rat growth, and distribution of proteins in sorghum. J. Agr. Food Chem. 21, 692-696 (1973).

41. Kumararaj, R.; Narahari, D.; Kothandaraman, P. Chemical composition and nutritive value of differently process tamarind seed meal in chick rations. Indian $J$. Poult. Sci. 16, 358-365 (1980).

42. Cousins, B.W.; Tanksley Jr., T.D.; Knabe, D.A.; Zebrowska, T. Nutrient digestibility and performance of pigs 
fed sorghums varying in tannin concentration. $J$. Anim. Sci. 53, 1524-1537 (1981).

43. Horigome, T.; Kumar, R.; Okamoto, K. Effects of condensed tannins prepared from leaves of fodder plants on digestive enzymes in vitro and in the intestine of rats. Brit. J. Nutr. 60, 275-285 (1988).

44. Featherston, W.R.; Rogler, J.C. Influence of tannins on the utilization of sorghum grain by rats and chicks. Nutr. Rep. International. 11, 491-497 (1975).

45. Al-Mamary, M.; Al-Habori, M.; Al-Aghbari, A.; Al-Obeidi, A. In vivo effects of dietary sorghum tannins on rabbit digestive enzymes and mineral absorption. Nutr Res. 21, 1393-401 (2001).

46. Sugiyama, H.; Yasue, M.; Kanda, T.; Shoji, T.; Ohtake, Y. Effects of polyphenols derived from apples on fat metabolism. 53-53 (2006).

47. Ohta, Y.; Funayama, M.; Seino, H.; Sami, M.; Kanda, T.; Shoji, T.; Ohtake, Y.; Osada, K. Apple polyphenols extract counteracts the development of obesity and impairment of glucose tolerance in zucker fatty rats. Food Sci. Tech. Res. (2007) in press.

48. Muramatsu, K.; Fukuyo, M.; Hara, Y. Effect of green tea catechins on plasma cholesterol level in cholesterol-fed rats. J. Nutr. Sci. Vitaminol. 32, 613-622 (1986).

49. Ikeda, I.; Imasato, Y.; Sasaki, E.; Nakayama, M.; Nagao, H.; Takeo, T.; Yayabe, F.; Sugano, M. Tea catechins decrease micellar solubility and intestinal absorption of cholesterol in rats. Biochim. Biophy. Acta - Lipids and Lipid Metabolism. 1127, 141-146 (1992).

50. Matsumoto, N.; Ishigaki, F.; Ishigaki, A.; Iwashina, H.; Hara, Y. Reduction of blood glucose levels by tea cate- chin. Biosci. Biotechnol. Biochem. 57, 525-527 (1993).

51. Meguro, S.; Mizuno, T.; Onizawa, K.; Kawasaki, K.; Nakagiri, H.; Hase, T.; Tokimitsu, I.; Shimasaki, H.; Itakura,H. Effects of tea catechins on diet-induced obesity in mice. J. Oleo Sci. 50, 593-598 (2001).

52. Dulloo, A.G.; Duret, C.; Rohrer, D.; Girardier, L.; Mensi, N.; Fathi, M.; Chantre, P.; Vandermander, J. Efficacy of a green tea extract rich in catechin polyphenols and caffeine in increasing 24-h energy expenditure and fat oxidation in humans. Am. J. Clin. Nutr. 70, 1040-1045 (1999).

53. Osaki, N.; Harada, U.; Watanabe, H.; Onizawa, K.; Yamaguchi, T.; Tokimitsu, I.; Shimasaki, H.; Itakura, H. Effect of tea catechins on energy metabolism in rats. $J$. Oleo Sci. 50, 677-682 (2001).

54. Ohta, Y.; Sami, M.; Kanda, T.; Saito, K.; Osada, K.; Kato, H. Gene expression analysis of the anti-obesity effect by apple polyphenols in rats fed a high fat diet or a normal diet. J. Oleo Sci. 55, 305-314 (2006).

55. Kondo, K.; Matsumoto, A.; Kurata, H.; Tanashashi, H.; Koda, H.; Amachi, T.; Itakura, H. Inhibition of oxidation of low-density lipoprotein with red wine [3]. Lancet. 344, 1152 (1994).

56. Nagao, T.; Meguro, S.; Soga, S.; Otsuka, A.; Tomonobu, K.; Fumoto, S.; Chikama, A.; Mori, K.; Yuzawa, M.; Watanabe, H.; Hase, T.; Tanaka, Y.; Tokimitsu, I.; Shimasaki, H.; Itakura, H. Tea catechins inhibit body fat manipulation in humans. J. Oleo Sci. 50, 717-728 (2001).

57. De Rozo, M.P.; Velez, J.; Garcia, L.A. Effect of the polyphenols of coffee pulp on iron absorption. Archivos Latinoamericanos de Nutricion. 35, 287-296 (1985). 